\title{
New Geometrical Spectra of Linear Codes with Applications to Performance Analysis
}

\author{
Xiao $\mathrm{Ma}^{*}$, Jia Liu*t, and Qiutao Zhuang* \\ ${ }^{*}$ Department of Electronics and Communication Engineering, Sun Yat-sen \\ University, Guangzhou 510006, GD, China \\ ${ }^{\dagger}$ College of Comp. Sci. and Eng., Zhongkai University of Agriculture and \\ Engineering, Guangzhou 510225, GD, China \\ Email:maxiao@mail.sysu.edu.cn, ljia2@mail2.sysu.edu.cn and \\ zhuangqt@mail2.sysu.edu.cn
}

\begin{abstract}
In this paper, new enumerating functions for linear codes are defined, including the triangle enumerating function and the tetrahedron enumerating function, both of which can be computed using a trellis-based algorithm over polynomial rings. The computational complexity is dominated by the complexity of the trellis. In addition, we show that these new enumerating functions can be used to improve existing performance bounds on the maximum likelihood decoding.
\end{abstract}

\section{INTRODUCTION}

The weight enumerating function (WEF) [1] is a figure of merit of a linear code, which plays a fundamental rule in the performance analysis of the maximum likelihood (ML) decoding algorithm. The conventional union bound, which involves only pair-wise error probabilities, is simple but loose and even diverges in the low signal-to-noise ratio (SNR) region. One general methodology to improve the conventional union bound, as shown in [2], is invoking the Gallager's first bounding technique (GFBT)

$$
\operatorname{Pr}\{E\} \leq \operatorname{Pr}\{E, \underline{y} \in \mathcal{R}\}+\operatorname{Pr}\{\underline{y} \notin \mathcal{R}\}
$$


where $E$ denotes the error event, $\underline{y}$ denotes the received signal vector, and $\mathcal{R}$ denotes an arbitrary region around the transmitted signal vector which is usually interpreted as the "good region". Most existing upper bounds within this framework, say, [3-10], first choose the region $\mathcal{R}$ such that the second term of (1) can be easily evaluated and then derive upper bounds on the first term of (1) by using (conditional) pair-wise error probabilities and the whole (or truncated) WEF of the code.

Yousefi and Khandani [9] derived an improved upper bound by using a Bonferroni-type inequality of the second degree instead of the union bound. Since the resulting upper bound cannot be calculated in terms of the distance spectrum of the code, the original codebook is enlarged by all $n$-tuples of Hamming weight $w$, resulting in a bound that is solely dependent on the distance spectrum but becomes looser. Very similarly, Ma et al [11] proposed using tripletwise error probabilities instead of pair-wise error probabilities to improve the union bound. To make the proposed bound computable in terms of the distance spectrum of the code, an upper bound on the triplet-wise error probability is derived in [11, Lemma 4]. It has been shown that the union bound based on the triplet-wise error probability is tighter than the conventional union bound [11, Theorem 1].

This paper is concerned with further tightening the union bound by alleviating the repeated accumulations caused by the use of the pair-wise error probabilities. The basic approach is to explore more detailed geometrical structure (beyond the distance spectrum) of the code when upper bounding the error probabilities. The main results as well as the structure of this paper are summarized as follows.

1) In Sec. II, we define two new enumerating functions for linear codes, the triangle spectrum and the tetrahedron spectrum, both of which can be calculated by a trellis-based algorithm.

2) In Sec. III, we derive improved union bounds based the triangle spectrum and the tetrahedron spectrum of binary linear codes. A toy example is given to show that the improvement is possible in the low-SNR region, as expected. The proposed union bound may be combined with other upper bounding techniques based on GFBT, potentially resulting in tighter upper bounds.

3) Sec. IV concludes this paper. 


\section{New Spectra of Linear Block Codes}

Let $\mathbb{F}_{q}$ be the finite field of size $q$. Let $\mathbb{F}_{q}^{n}$ denote the $n$-dimensional vector space consisting of $n$-tuples over $\mathbb{F}_{q}$. Given $\underline{v} \triangleq\left(v_{0}, v_{1}, \cdots, v_{n-1}\right) \in \mathbb{F}_{q}^{n}$, the number of non-zero component of $\underline{v}$, denoted by $W_{H}(\underline{v})$, is called the Hamming weight of $\underline{v}$. The Hamming distance between two vectors $\underline{v}$ and $\underline{w}$ is defined as $W_{H}(\underline{w}-\underline{v})$. A linear code $\mathcal{C}_{q}[n, k]$ is defined as a $k$-dimensional linear subspace of $\mathbb{F}_{q}^{n}$. A vector in $\mathcal{C}_{q}[n, k]$ is called a codeword. There are $q^{k}$ in total codewords in $\mathcal{C}_{q}[n, k]$, which are simply indexed by $\underline{c}^{(i)}, 0 \leq i \leq q^{k}-1$. Specifically, we use $\underline{c}^{(0)}$ to represent the all-zero codeword.

\section{A. Weight Enumerating Function}

Definition 1: The weight enumerating function $(W E F)$ of $\mathcal{C}_{q}[n, k]$ is defined as [1]

$$
A(X) \triangleq \sum_{i} A_{i} X^{i}
$$

where $X$ is a dummy variable and $A_{i}$ denotes the number of codewords having Hamming weight $i$.

The sequence $\left\{A_{i}, 0 \leq i \leq n\right\}$ is also called weight spectrum of the code, which exhibits how many codewords that are $i$ positions far away from the reference codeword $\underline{c}^{(0)}$. By linearity, we know that the weight spectrum is irrelevant to the reference codeword. Clearly, we have

$$
\sum_{1 \leq i \leq n} A_{i}=q^{k}-1
$$

For a binary code with the all-one codeword, we further have $A_{i}=A_{n-i}$ for $0 \leq i \leq n$.

\section{B. Triangle Enumerating Function}

Definition 2: Let $\underline{c}^{(0)}$ be the all-zero codeword and $\underline{c}^{(1)}$ be an arbitrarily given non-zero codeword. The triangle enumerating function $(\operatorname{TrEF})$ of $\mathcal{C}_{q}[n, k]$ is defined as

$$
B\left(\underline{c}^{(1)} ; X, Y\right) \triangleq \sum_{i, j} B_{i, j}\left(\underline{c}^{(1)}\right) X^{i} Y^{j},
$$

where $X, Y$ are two dummy variables and $B_{i, j}\left(\underline{c}^{(1)}\right)$ denotes the number codewords $\underline{c}$ satisfying $W_{H}\left(\underline{c}-\underline{c}^{(0)}\right)=i$ and $W_{H}\left(\underline{c}-\underline{c}^{(1)}\right)=j$. 
Generally, the TrEF depends on the choice of the reference codeword $\underline{c}^{(1)}$. When the context is clear, we may drop the reference codeword from the notation. The sequence $\left\{B_{i, j}, 0 \leq i, j \leq n\right\}$ is also called the triangle spectrum of the code. Clearly, we have

$$
\sum_{1 \leq i, j \leq n} B_{i, j}=q^{k}-2
$$

For binary codes with the all-one codeword, we have the following proposition.

Proposition 1: Suppose that $\mathcal{C}_{2}[n, k]$ has the WEF $A(X)$ such that $A_{n}=1$. Let $\underline{c}^{(1)}$ be the codeword of weight $n$. Then

$$
B_{i, j}=\left\{\begin{array}{cc}
A_{i}, & i+j=n \\
0, & i+j \neq n
\end{array} .\right.
$$

Proof: It can be proved by noticing that $W_{H}\left(\underline{c}-\underline{c}^{(0)}\right)=i$ if and only if $W_{H}\left(\underline{c}-\underline{c}^{(1)}\right)=n-i$.

\section{Tetrahedron Enumerating Function}

Definition 3: Let $\underline{c}^{(0)}$ be the all-zero codeword. Let $\underline{c}^{(1)}$ and $\underline{c}^{(2)}$ be two arbitrarily given codewords. The tetrahedron enumerating function $(T e E F)$ of the code $\mathcal{C}_{q}[n, k]$ is defined as

$$
C\left(\underline{c}^{(1)}, \underline{c}^{(2)} ; X, Y, Z\right) \triangleq \sum_{i, j, h} C_{i, j, h}\left(\underline{c}^{(1)}, \underline{c}^{(2)}\right) X^{i} Y^{j} Z^{h},
$$

where $X, Y, Z$ are three dummy variables and $C_{i, j, h}\left(\underline{c}^{(1)}, \underline{c}^{(2)}\right)$ denotes the number of codewords $\underline{c}$ satisfying $W_{H}\left(\underline{c}-\underline{c}^{(0)}\right)=i, W_{H}\left(\underline{c}-\underline{c}^{(1)}\right)=j$ and $W_{H}\left(\underline{c}-\underline{c}^{(2)}\right)=h$.

Generally, the TeEF depends on the choice of the reference codewords $\underline{c}^{(1)}$ and $\underline{c}^{(2)}$. When the context is clear, we may drop the reference codewords from the notation. The sequence $\left\{C_{i, j, h}, 0 \leq i, j, h \leq n\right\}$ is also called the tetrahedron spectrum of the code. Clearly, we have

$$
\sum_{1 \leq i, j, h \leq n} C_{i, j, h}=q^{k}-3
$$

\section{An Example}

We take the Hamming code $\mathcal{C}_{2}[7,4]$ as an example to illustrate the introduced enumerating functions.

The WEF is

$$
A(X)=1+7 X^{3}+7 X^{4}+X^{7}
$$


Since the TrEF depends on the choice of the reference codeword $\underline{c}^{(1)}$, we distinguish following three cases.

Case 1: If $W_{H}\left(\underline{c}^{(1)}\right)=7$,

$$
B(X, Y)=Y^{7}+7 X^{3} Y^{4}+7 X^{4} Y^{3}+X^{7} .
$$

Case 2: If $W_{H}\left(\underline{c}^{(1)}\right)=4$,

$$
B(X, Y)=Y^{4}+X^{4}+6 X^{3} Y^{3}+X^{3} Y^{7}+6 X^{4} Y^{4}+X^{7} Y^{3} .
$$

Case 3: If $W_{H}\left(\underline{c}^{(1)}\right)=3$,

$$
B(X, Y)=Y^{3}+X^{3}+6 X^{3} Y^{4}+6 X^{4} Y^{3}+X^{4} Y^{7}+X^{7} Y^{4} .
$$

Similarly, the TeEF also depends on the choices of the reference codewords $\underline{c}^{(1)}$ and $\underline{c}^{(2)}$. We have

Case 1: If $W_{H}\left(\underline{c}^{(1)}\right)=3$ and $W_{H}\left(\underline{c}^{(2)}\right)=3$,

$$
\begin{aligned}
& C(X, Y, Z)=Y^{3} Z^{3}+X^{3} Y^{4}+X^{3} Z^{4}+ \\
& 5 X^{3} Y^{4} Z^{4}+5 X^{4} Y^{3} Z^{3}+X^{4} Y^{3} Z^{7}+X^{4} Y^{7} Z^{3}+X^{7} Y^{4} Z^{4} .
\end{aligned}
$$

Case 2: If $W_{H}\left(\underline{c}^{(1)}\right)=3, W_{H}\left(\underline{c}^{(2)}\right)=4$, and $W_{H}\left(\underline{c}^{(2)}-\underline{c}^{(1)}\right)=3$,

$$
\begin{aligned}
& C(X, Y, Z)=Y^{3} Z^{4}+X^{3} Z^{3}+X^{4} Y^{3}+ \\
& 5 X^{3} Y^{4} Z^{3}+X^{3} Y^{4} Z^{7}+5 X^{4} Y^{3} Z^{4}+X^{4} Y^{7} Z^{4}+X^{7} Y^{4} Z^{3} .
\end{aligned}
$$

Case 2': If $W_{H}\left(\underline{c}^{(1)}\right)=3, W_{H}\left(\underline{c}^{(2)}\right)=4$, and $W_{H}\left(\underline{c}^{(2)}-\underline{c}^{(1)}\right)=7$,

$$
\begin{aligned}
& C(X, Y, Z)=Y^{3} Z^{4}+X^{3} Z^{7}+X^{4} Y^{7}+ \\
& 6 X^{3} Y^{4} Z^{3}+6 X^{4} Y^{3} Z^{4}+X^{7} Y^{4} Z^{3} .
\end{aligned}
$$

Case 3: If $W_{H}\left(\underline{c}^{(1)}\right)=3$ and $W_{H}\left(\underline{c}^{(2)}\right)=7$,

$$
\begin{aligned}
& C(X, Y, Z)=Y^{3} Z^{7}+X^{3} Z^{4}+X^{7} Y^{4}+ \\
& 6 X^{3} Y^{4} Z^{4}+6 X^{4} Y^{3} Z^{3}+X^{4} Y^{7} Z^{3}
\end{aligned}
$$

Case 4: If $W_{H}\left(\underline{c}^{(1)}\right)=4$ and $W_{H}\left(\underline{c}^{(2)}\right)=4$,

$$
\begin{aligned}
& C(X, Y, Z)=Y^{4} Z^{4}+X^{4} Z^{4}+X^{4} Y^{4}+ \\
& 5 X^{3} Y^{3} Z^{3}+X^{3} Y^{3} Z^{7}+X^{3} Y^{7} Z^{3}+5 X^{4} Y^{4} Z^{4}+X^{7} Y^{3} Z^{3} .
\end{aligned}
$$


Case 5: If $W_{H}\left(\underline{c}^{(1)}\right)=4$ and $W_{H}\left(\underline{c}^{(2)}\right)=7$,

$$
\begin{aligned}
& C(X, Y, Z)=Y^{4} Z^{7}+X^{4} Z^{3}+X^{7} Y^{3}+ \\
& 6 X^{3} Y^{3} Z^{4}+X^{3} Y^{7} Z^{4}+6 X^{4} Y^{4} Z^{3}
\end{aligned}
$$

\section{E. Computing the Enumerating Functions Over a Trellis}

It is well-known that any linear block code can be represented by a trellis [12] [13]. Generally, a trellis that represents $\mathcal{C}_{q}[n, k]$ can have $N$ stages. The trellis section at stage $t(0 \leq t \leq N-1)$, denoted by $\mathcal{B}_{t}$, is a subset of $\mathcal{S}_{t} \times \mathbb{F}_{q}^{n_{t}} \times \mathcal{S}_{t+1}$, where $\mathcal{S}_{t}$ is the state space at time $t$. A branch $b \in \mathcal{B}_{t}$ is denoted by $b \triangleq\left(\sigma^{-}(b), \ell(b), \sigma^{+}(b)\right)$, starting from a state $\sigma^{-}(b) \in \mathcal{S}_{t}$, taking a label $\ell(b) \in \mathbb{F}_{q}^{n_{t}}$, and ending into a state $\sigma^{+}(b) \in \mathcal{S}_{t+1}$. A path through a trellis is a sequence of branches $\underline{b}=$ $\left(b_{0}, b_{1}, \cdots, b_{N-1}\right)$ satisfying that $b_{t} \in \mathcal{B}_{t}$ and $\sigma^{-}\left(b_{t+1}\right)=\sigma^{+}\left(b_{t}\right)$. A codeword is then represented by a path in the sense that $\underline{c}=\left(\ell\left(b_{0}\right), \ell\left(b_{1}\right), \cdots, \ell\left(b_{N-1}\right)\right)$. Naturally, $\sum_{0 \leq t \leq N-1} n_{t}=n$ and the number of paths is $q^{k}$. Without loss of generality, we set $\mathcal{S}_{0}=\mathcal{S}_{N}=\{0\}$.

Proposition 2: Given a trellis representation of $\mathcal{C}_{q}[n, k]$. Let $\underline{c}^{(0)}$ (the all-zero codeword), $\underline{c}^{(1)}$ and $\underline{c}^{(2)}$ be three reference codewords. The corresponding pathes are denoted by $\underline{b}^{(0)}=$ $\left(b_{0}^{(0)}, b_{1}^{(0)}, \cdots, b_{N-1}^{(0)}\right), \underline{b}^{(1)}=\left(b_{0}^{(1)}, b_{1}^{(1)}, \cdots, b_{N-1}^{(1)}\right)$ and $\underline{\underline{b}}^{(2)}=\left(b_{0}^{(2)}, b_{1}^{(2)}, \cdots, b_{N-1}^{(2)}\right)$, respectively. Then the enumerating function (WEF, TrEF or TeEF) is equal to $\alpha_{N}(0)$, as calculated recursively by the following trellis-based algorithm over a properly defined polynomial ring.

- Initially, set $\alpha_{0}(0)=1$.

- For $t=0,1, \cdots, N-1$,

$$
\alpha_{t+1}(s)=\sum_{b \in \mathcal{B}_{t}, \sigma^{+}(b)=s} \alpha_{t}\left(\sigma^{-}(b)\right) \gamma_{t}(b)
$$

for each state $s \in \mathcal{S}_{t+1}$, where $\gamma_{t}(b)$ is specified as follows.

Case 1: For computing WEF, $\gamma_{t}(b) \triangleq X^{i}$, where $i=W_{H}(\ell(b))$.

Case 2: For computing TrEF, $\gamma_{t}(b) \triangleq X^{i} Y^{j}$, where $i=W_{H}(\ell(b))$ and $j=W_{H}(\ell(b)-$ $\left.\ell\left(b_{t}^{(1)}\right)\right)$.

Case 3: For computing TeEF, $\gamma_{t}(b) \triangleq X^{i} Y^{j} Z^{h}$, where $i=W_{H}(\ell(b)), j=W_{H}(\ell(b)-$ $\left.\ell\left(b_{t}^{(1)}\right)\right)$ and $h=W_{H}\left(\ell(b)-\ell\left(b_{t}^{(2)}\right)\right)$.

Proof: The algorithm is similar to the trellis algorithm over polynomial rings for computing the weight enumerators of paths [12]. 
Remark. It can be seen that the computational complexity of the algorithm given in Proposition 2 is dominated by the complexity of the trellis [12]. From this algorithm, we also know that $B\left(\underline{c}^{(1)} ; X, Y\right)=C\left(\underline{c}^{(1)}, \underline{c}^{(2)} ; X, Y, Z=1\right)$ and $A(X)=B\left(\underline{c}^{(1)} ; X, Y=1\right)$.

\section{IMPROVED UNION BOUNDS FOR BINARY LINEAR CODES BASED ON GEOMETRICAL SPECTRA}

In this section, we focus on tightening the conventional union bound based on pair-wise error probabilities by exploring further the geometrical structure of codes.

\section{A. Geometrical Properties of Binary Codes}

Let $\mathbb{F}_{2}=\{0,1\}$ and $\mathcal{A}_{2}=\{-1,+1\}$ be the binary field and the bipolar signal set, respectively. Suppose that a codeword $\underline{c}=\left(c_{0}, c_{1}, \cdots, c_{n-1}\right) \in \mathcal{C}_{2}[n, k]$ is modulated by binary phase shift keying (BPSK), resulting in a bipolar signal vector $\underline{s} \in \mathcal{A}_{2}^{n}$ with $s_{t}=1-2 c_{t}$ for $0 \leq t \leq n-1$. We will not distinguish between a binary codeword $\underline{c}$ and its bipolar image in the following, except when we need to emphasize the difference between the Hamming space $\mathbb{F}_{2}^{n}$ and the Euclidean space $\mathbb{R}^{n} \supset \mathcal{A}_{2}^{n}$. The Euclidean distance between two codewords $\underline{s}^{(1)}$ and $\underline{s}^{(2)}$ is related to their Hamming distance by $\left\|\underline{s}^{(2)}-\underline{s}^{(1)}\right\|=2 \sqrt{W_{H}\left(\underline{c}^{(2)}-\underline{c}^{(1)}\right)}$. All codewords are distributed on the surface of an $n$-dimensional sphere centered at the origin with radius $\sqrt{n}$. This property is referred to as the sphericity of the bipolar code.

Assume that a codeword $\underline{s}$ is transmitted over an AWGN channel, resulting in a received vector $\underline{y}=\underline{s}+\underline{z}$, where $\underline{z}$ is a sample from a white Gaussian noise process with zero mean and doublesided power spectral density $\sigma^{2}$. The ML decoding is equivalent to finding a bipolar codeword $\underline{s}$ that is the closest to $\underline{y}$. Since the decoding metric is the Euclidean distance, the geometrical structure of the code in $\mathbb{R}^{n}$ is supposed to be critical to analyze the ML decoding performance. However, to the best knowledge of ours, with the exception of the distance spectrum and the sphericity of the code, other figures of merits of the code were rarely employed to upper bound the ML decoding error probability. To reveal more information about the geometrical structure of the code, we have the following two propositions, where Proposition 3 was originally mentioned in [14] without proofs.

Proposition 3: Any three codewords form a non-obtuse triangle. Furthermore, if some three codewords form a right angle, there must exist a fourth codeword completing the rectangle. 
Proof: For a detailed proof of the first part, see [11].

To prove the second part, we may assume by linearity that $\underline{s}^{(0)}, \underline{s}^{(1)}$ and $\underline{s}^{(2)}$ form a right angle, that is, $\underline{s}^{(0)} \underline{s}^{(1)}$ is orthogonal to $\underline{s}^{(0)} \underline{s}^{(2)}$. Noting that this holds if and only if $W_{H}\left(\underline{c}^{(1)}+\underline{c}^{(2)}\right)=$ $W_{H}\left(\underline{c}^{(1)}\right)+W_{H}\left(\underline{c}^{(2)}\right)$, implying that the two codewords $\underline{c}^{(1)}$ and $\underline{c}^{(2)}$ are not "overlapped" (no common non-zero positions). Hence the binary addition $\underline{c}^{(1)}+\underline{c}^{(2)}$ can be treated as a real addition. Define the codeword $\underline{c}^{(3)}=\underline{c}^{(1)}+\underline{c}^{(2)}$. We can verify that

$$
\underline{s}^{(3)}-\underline{s}^{(0)}=\underline{s}^{(1)}-\underline{s}^{(0)}+\underline{s}^{(2)}-\underline{s}^{(0)},
$$

which means that $\underline{s}^{(0)} \underline{s}^{(3)}$ falls inside the plane determined by $\underline{s}^{(0)} \underline{s}^{(1)}$ and $\underline{s}^{(0)} \underline{s}^{(2)}$ and hence $\underline{s}^{(0)}$, $\underline{s}^{(1)}, \underline{s}^{(2)}$ and $\underline{s}^{(3)}$ must form a rectangle. Otherwise, some three of them would form an obtuse triangle.

Proposition 4: Any four codewords form either a tetrahedron or a rectangle.

Proof: From Proposition 3, any three codewords form a non-obtuse triangle, which determines a two-dimensional plane. If the fourth codeword falls inside the same plane, the four codewords must form a rectangle; otherwise, some three of them would form an obtuse triangle. If the fourth codeword falls outside that plane, then the four codewords form a tetrahedron in a three-dimensional space.

With BPSK signalling, we also refer WEF, TrEF and TeEF to as geometrical spectra of a code. Fig. 1 shows the geometrical spectra of the Hamming code $\mathcal{C}_{2}[7,4]$.

\section{B. Improved Union Bounds Based on Geometrical Spectra}

Assume that $\underline{s}^{(0)}$ is transmitted. For a codeword $\underline{s}$, let

$$
\left\{\underline{s}^{(0)} \rightarrow \underline{s}\right\} \triangleq\left\{\underline{y}:\|\underline{y}-\underline{s}\| \leq\left\|\underline{y}-\underline{s}^{(0)}\right\|\right\}
$$

which is the event that $\underline{s}$ is nearer than $\underline{s}^{(0)}$ to $\underline{y}$. We use $\left\{\underline{s}^{(0)} \nrightarrow \underline{s}\right\}$ to denote the complementary event.

To derive the upper bounds on the decoding error probability $\operatorname{Pr}\{E\}$, we take two arbitrary but fixed codewords $\underline{s}^{(1)}$ and $\underline{s}^{(2)}$ as reference codewords. Let $d_{1}=W_{H}\left(\underline{c}^{(1)}\right), d_{2}=W_{H}\left(\underline{c}^{(2)}\right)$ and $d_{1,2}=W_{H}\left(\underline{c}^{(1)}-\underline{c}^{(2)}\right)$. For a codeword $\underline{c}$, let $i=W_{H}\left(\underline{c}-\underline{c}^{(0)}\right), j=W_{H}\left(\underline{c}-\underline{c}^{(1)}\right)$ and $h=$ $W_{H}\left(\underline{c}-\underline{c}^{(2)}\right)$. It is well-known that the pair-wise error probability $(P E P) p_{2}(i) \triangleq \operatorname{Pr}\left\{\underline{s}^{(0)} \rightarrow \underline{s}\right\}$ 
(a)
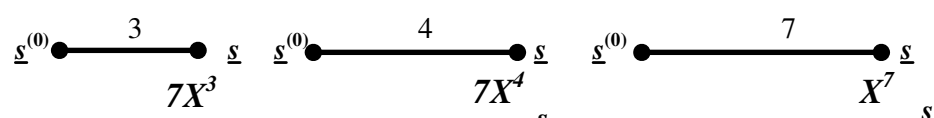

(b)

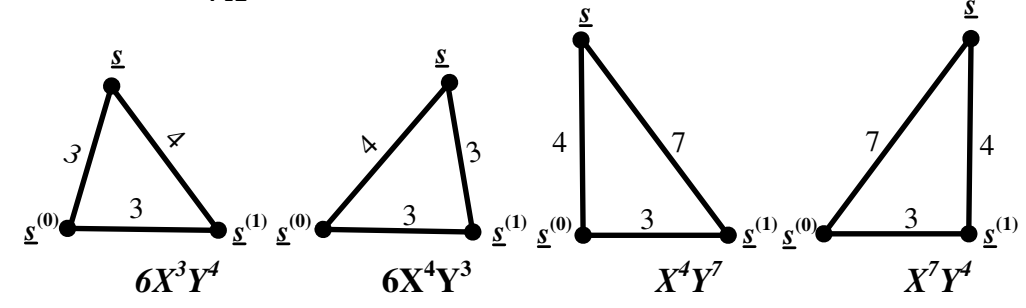

(c)
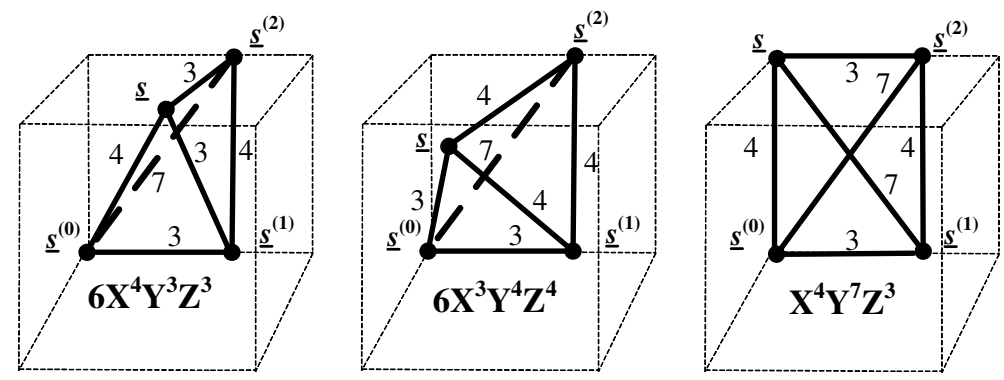

Fig. 1. Geometrical spectra of the Hamming code $\mathcal{C}_{2}[7,4]$, where $\underline{s}^{(i)}, i=0,1,2$, are taken as the reference codewords and each edge is labeled by the Hamming distance. (a) The weight spectrum. (b) A triangle spectrum. (c) A tetrahedron spectrum.

is given by $Q(\sqrt{i} / \sigma)$ and depends solely on the Hamming weight. Going a step further, we can verify that the triplet-wise error probability $(\operatorname{TrEP})$, defined by

$$
p_{3}(i, j) \triangleq \operatorname{Pr}\left\{\left(\underline{s}^{(0)} \rightarrow \underline{s}^{(1)}\right) \bigcup\left(\underline{s}^{(0)} \rightarrow \underline{s}\right)\right\},
$$

depends solely on the triangle formed by the three codewords. Similarly, the quadruple-wise error probability $(Q u E P)$, defined by

$$
p_{4}(i, j, h) \triangleq \operatorname{Pr}\left\{\left(\underline{s}^{(0)} \rightarrow \underline{s}^{(1)}\right) \bigcup\left(\underline{s}^{(0)} \rightarrow \underline{s}^{(2)}\right) \bigcup\left(\underline{s}^{(0)} \rightarrow \underline{s}\right)\right\},
$$

depends solely on the tetrahedron (or rectangle) formed by the four codewords. For these reasons, we have dropped the codeword $\underline{s}$ from the notation and simply denoted these probabilities by $p_{2}(i), p_{3}(i, j)$ and $p_{4}(i, j, h)$ as shown above.

To compute the introduced error probabilities conveniently, we may use a new coordinate system by choosing $\underline{s}^{(0)}$ as the origin $O$ and taking $\underline{s}^{(0)} \underline{s}^{(1)}$ as an axis, denoted by $\xi_{1}$-coordinate. We further choose $\xi_{2}$-coordinate such that $\underline{s}^{(2)}$ falls into the first quadrant of the plane $\xi_{1} O \xi_{2}$. Similarly, we choose $\xi_{3}$-coordinate such that the fourth codeword $\underline{s}$ falls in the first octant, as shown in Fig. 2. Note that such an arrangement does not lose any generality. Let $Z_{\xi_{1}}, Z_{\xi_{2}}$, and $Z_{\xi_{3}}$ be the three components obtained by projecting the noise $\underline{Z}$ onto the three axes, respectively. 


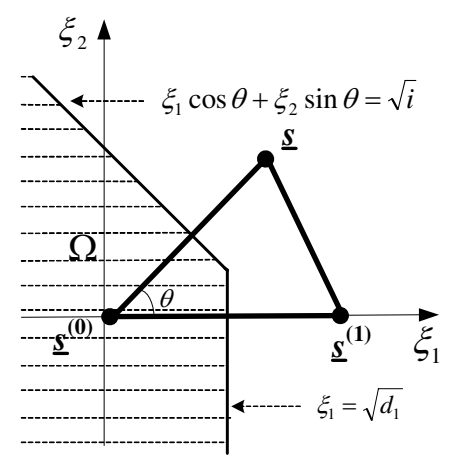

(a)

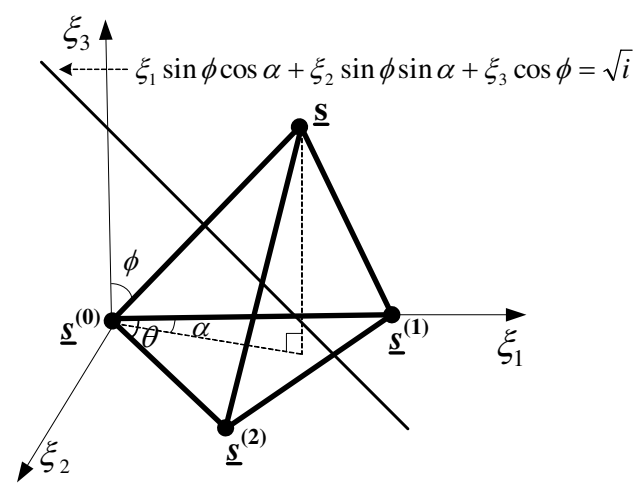

(b)

Fig. 2. Geometrical interpretation of error probabilities. (a) Triplet-wise error probability. (b) Quadruple-wise error probability.

Specifically, $Z_{\xi_{1}}$ is the inner product $\left\langle\underline{Z}, \frac{\underline{s}^{(1)}-\underline{s}^{(0)}}{\left\|\underline{s}^{(1)}-\underline{s}^{(0)}\right\|}\right\rangle$. These three component are independent and identically distributed as a Gaussian random variable with a probability density function $f(x)=\frac{1}{\sqrt{2 \pi} \sigma} \exp \left\{-\frac{x^{2}}{2 \sigma^{2}}\right\}$. We have the following lemmas.

Lemma 1: The TrEP can be calculated as

$$
p_{3}(i, j)=1-\iint_{\Omega} f\left(\xi_{1}\right) f\left(\xi_{2}\right) \mathrm{d} \xi_{1} \mathrm{~d} \xi_{2},
$$

where $\Omega=\left\{\xi_{1}<\sqrt{d_{1}}, \xi_{1} \cos \theta+\xi_{2} \sin \theta<\sqrt{i}\right\}$ and $\cos \theta=\left(d_{1}+i-j\right) /\left(2 \sqrt{d_{1} i}\right)$.

Proof: It can be proved by verifying that, given the three codewords, $\Omega$ is exactly the Voronoi region of $\underline{s}^{(0)}$. See Fig. 2 (a) for a reference.

Lemma 2: The QuEP can be calculated as

$$
p_{4}(i, j, h)=1-\iiint_{\Omega} f\left(\xi_{1}\right) f\left(\xi_{2}\right) f\left(\xi_{3}\right) \mathrm{d} \xi_{1} \mathrm{~d} \xi_{2} \mathrm{~d} \xi_{3} .
$$

The integration domain

$$
\Omega=\left\{\begin{array}{l}
\xi_{1}<\sqrt{d_{1}}, \quad \xi_{1} \cos \theta+\xi_{2} \sin \theta<\sqrt{d_{2}}, \\
\xi_{1} \sin \phi \cos \alpha+\xi_{2} \sin \phi \sin \alpha+\xi_{3} \cos \phi<\sqrt{i}
\end{array}\right\}
$$

can be determined by computing the azimuth angle $\theta$ of $\underline{s}^{(2)}$, the azimuth angle $\alpha$ of $\underline{s}$ and the colatitude angle $\phi$ of $\underline{s}$. See Fig. 2 (b) for a reference.

Proof: It can be proved by verifying that, given the four codewords, $\Omega$ is exactly the Voronoi region of $\underline{s}^{(0)}$. 
Remark. Note that the angles appeared in Lemma 2 are computable given the edge lengths of the tetrahedron. For example, $\theta$ can be computed by the law of cosines: $\cos \theta=\left(d_{1}+d_{2}-\right.$ $\left.d_{1,2}\right) /\left(2 \sqrt{d_{1} d_{2}}\right)$. And the expressions for $\alpha$ and $\phi$ can be obtained by

$$
\alpha=\arctan \left(\frac{\sqrt{\left(i+d_{1}-j\right)^{2} \cos ^{2} \theta+\frac{d_{1}\left(i+d_{2}-h\right)^{2}}{d_{2}}-\frac{2 \sqrt{d_{1} \cos \theta\left(i+d_{1}-j\right)\left(i+d_{2}-h\right)}}{\sqrt{d_{2}}}}}{\left(i+d_{1}-j\right) \sin \theta}\right)
$$

and

$$
\phi=\arcsin \left(\frac{\sqrt{4 i \sin ^{2} \theta-\frac{\left(i+d_{1}-j\right)^{2}}{d_{1}}-\frac{\left(i+d_{2}-h\right)^{2}}{d_{2}}+\frac{2 \cos \theta\left(i+d_{1}-j\right)\left(i+d_{2}-h\right)}{\sqrt{d_{1} d_{2}}}}}{2 \sqrt{i} \sin \theta}\right),
$$

respectively.

Also note that Lemma 2 is still valid in the case when the four codewords form a rectangle. It is worth pointing out the both TrEP and QuEP can be transformed into repeated integrals easily.

Theorem 1: Let $\underline{c}^{(1)}$ be any fixed reference codeword with $W_{H}\left(\underline{c}^{(1)}\right)=d_{1} \geq 1$. Assume that the corresponding triangle spectrum $\left\{B_{i, j}\right\}$ is available. The ML decoding error can be upper bounded by

$$
\operatorname{Pr}\{E\} \leq-\left(2^{k}-3\right) Q\left(\sqrt{d_{1}} / \sigma\right)+\sum_{1 \leq i, j \leq n} B_{i, j} p_{3}(i, j),
$$

where $p_{3}(i, j)$ are given by (10).

Proof: From the second-order Bonferroni-type inequality, we have

$$
\begin{aligned}
& \operatorname{Pr}\{E\}=\operatorname{Pr}\left\{\bigcup_{\underline{s} \neq \underline{s}^{(0)}}\left(\underline{s}^{(0)} \rightarrow \underline{s}\right)\right\} \\
& \leq \operatorname{Pr}\left\{\underline{s}^{(0)} \rightarrow \underline{s}^{(1)}\right\}+\sum^{\prime} \operatorname{Pr}\left\{\underline{s}^{(0)} \nrightarrow \underline{s}^{(1)}, \underline{s}^{(0)} \rightarrow \underline{s}\right\} \\
& =-\left(2^{k}-3\right) \operatorname{Pr}\left\{\underline{s}^{(0)} \rightarrow \underline{s}^{(1)}\right\}+\sum^{\prime} \operatorname{Pr}\left\{\left(\underline{s}^{(0)} \rightarrow \underline{s}^{(1)}\right) \bigcup\left(\underline{s}^{(0)} \rightarrow \underline{s}\right)\right\},
\end{aligned}
$$

where the summation $\sum^{\prime}$ is over all $\left\{\underline{s}: \underline{s} \neq \underline{s}^{(0)}, \underline{s} \neq \underline{s}^{(1)}\right\}$. This completes the proof by noting that the TrEP depends only on the types of the triangles.

Theorem 2: Let $\underline{c}^{(1)}$ and $\underline{c}^{(2)}$ be any two fixed reference codewords with $W_{H}\left(\underline{c}^{(1)}\right)=d_{1} \geq 1$, $W_{H}\left(\underline{c}^{(2)}\right)=d_{2} \geq 1$ and $W_{H}\left(\underline{c}^{(2)}-\underline{c}^{(1)}\right)=d_{1,2} \geq 1$. Assume that the corresponding tetrahedron spectrum $\left\{C_{i, j, h}\right\}$ is available. The ML decoding error can be upper bounded by

$$
\operatorname{Pr}\{E\} \leq-\left(2^{k}-4\right) p_{3}\left(d_{2}, d_{1,2}\right)+\sum_{1 \leq i, j, h \leq n} C_{i, j, h} p_{4}(i, j, h),
$$




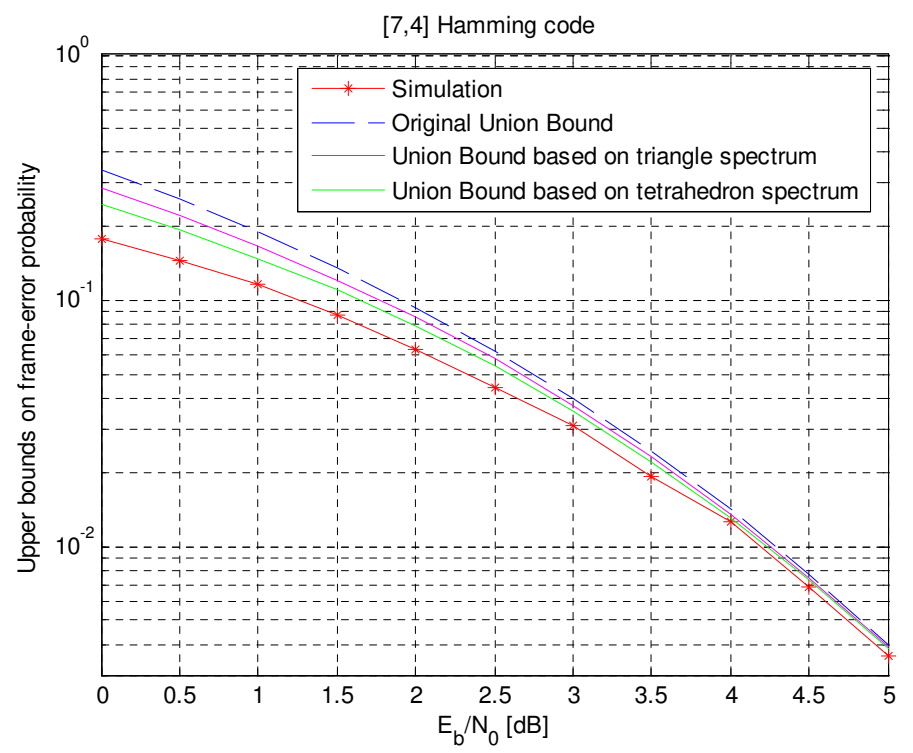

Fig. 3. Comparison between the upper bounds on the frame-error probability under ML decoding of [7, 4] Hamming code. The compared bounds are the original union bound, the union bound based on triangle spectrum and the union bound based on tetrahedron spectrum, which are also compared with the ML simulation results.

where $p_{3}\left(d_{2}, d_{1,2}\right)$ and $p_{4}(i, j, h)$ are given by (10) and (11), respectively.

Proof: From the third-order Bonferroni-type inequality, we have

$$
\begin{aligned}
& \operatorname{Pr}\{E\}=\operatorname{Pr}\left\{{\underline{\underline{s}} \neq \underline{s}^{(0)}}\left(\underline{s}^{(0)} \rightarrow \underline{s}\right)\right\} \\
& \leq \operatorname{Pr}\left\{\left(\underline{s}^{(0)} \rightarrow \underline{s}^{(1)}\right) \bigcup\left(\underline{s}^{(0)} \rightarrow \underline{s}^{(2)}\right)\right\}+ \\
& \quad \sum_{\underline{s} \neq \underline{s}^{(i)}, i=0,1,2} \operatorname{Pr}\left\{\underline{s}^{(0)} \nrightarrow \underline{s}^{(1)}, \underline{s}^{(0)} \nrightarrow \underline{s}^{(2)}, \underline{s}^{(0)} \rightarrow \underline{s}\right\} \\
& =-\left(2^{k}-4\right) \operatorname{Pr}\left\{\left(\underline{s}^{(0)} \rightarrow \underline{s}^{(1)}\right) \bigcup\left(\underline{s}^{(0)} \rightarrow \underline{s}^{(2)}\right)\right\} \\
& +\sum_{\underline{s}^{\prime} \underline{s}^{(i)}, i=0,1,2} \operatorname{Pr}\left\{\left(\underline{s}^{(0)} \rightarrow \underline{s}^{(1)}\right) \bigcup\left(\underline{s}^{(0)} \rightarrow \underline{s}^{(2)}\right) \bigcup\left(\underline{s}^{(0)} \rightarrow \underline{s}\right)\right\},
\end{aligned}
$$

completing the proof.

\section{Numerical Results}

From the proofs of Theorems 1 and 2, we know that the proposed bounds compute the higherorder Bonferroni-type inequalities. Hence the proposed bounds are tighter than the conventional union bound. To verify this numerically, we give an example. Fig. 3 shows the comparisons 
between the original union bound and the bounds given in Theorems 1 and 2 on the frameerror probability of the Hamming code $\mathcal{C}_{2}[7,4]$. Also shown are the simulation results. The TrEF and TeEF we choose are $B(X, Y)=Y^{3}+X^{3}+6 X^{3} Y^{4}+6 X^{4} Y^{3}+X^{4} Y^{7}+X^{7} Y^{4}$ and $C(X, Y, Z)=Y^{3} Z^{3}+X^{3} Y^{4}+X^{3} Z^{4}+5 X^{3} Y^{4} Z^{4}+5 X^{4} Y^{3} Z^{3}+X^{4} Y^{3} Z^{7}+X^{4} Y^{7} Z^{3}+X^{7} Y^{4} Z^{4}$, respectively. We can see that the bounds using higher-order Bonferroni-type inequalities are tighter, as expected.

\section{CONCLUSIONS}

In this paper, we have presented the definition of TrEF and TeEF, both of which can be computed using a trellis-based algorithm over polynomial rings. We have also derived the upper bounds based on triangle spectrum and tetrahedron spectrum, respectively, which can be used to improve the union bound by alleviating the repeated accumulations caused by the use of the pair-wise error probabilities.

\section{REFERENCES}

[1] F. J. MacWilliams and N. J. A. Sloane, The theory of error correcting codes. New York: North Holland Publishing Co., 1977.

[2] I. Sason and S. Shamai, "Performance analysis of linear codes under maximum-likelihood decoding: A tutorial," in Foundations and Trends in Communications and Information Theory. Delft, The Netherlands: NOW, July 2006, vol. 3, no. 1-2, pp. 1-225.

[3] E. R. Berlekamp, “The technology of error correction codes," Proceedings of the IEEE, vol. 68, pp. 564-593, May 1980.

[4] T. Kasami, T. Fujiwara, T. Takata, K. Tomita, and S. Lin, "Evaluation of the block error probability of block modulation codes by the maximum-likelihood decoding for an AWGN channel," in Proc. of the 15th Symposium on Information Theory and Its Applications, Minakami, Japan, September 1992.

[5] H. Herzberg and G. Poltyrev, "Techniques of bounding the probability of decoding error for block coded modulation structures," IEEE Transactions on Information Theory, vol. 40, pp. 903-911, May 1994.

[6] G. Poltyrev, "Bounds on the decoding error probability of binary linear codes via their spectra," IEEE Transactions on Information Theory, vol. 40, pp. 1284-1292, July 1994.

[7] J. Zangl and R. Herzog, "Improved tangential sphere bound on the bit error probability of concatenated codes," IEEE Journal on Selected Areas in Communications, vol. 19, pp. 825-830, May 2001.

[8] D. Divsalar and E. Biglieri, "Upper bounds to error probabilities of coded systems beyond the cutoff rate," IEEE Trans. Commun., vol. 51, no. 12, pp. 2011-2018, December 2003. 
[9] S. Yousefi and A. K. Khandani, "A new upper bound on the ML decoding error probability of linear binary block codes in AWGN interference," IEEE Transactions on Information Theory, vol. 50, pp. 3026-3036, Novomber 2004.

[10] A. Mehrabian and S. Yousefi, "Improved tangential sphere bound on the ML decoding error probability of linear binary block codes in AWGN and block fading channels," IEE Proc. Commun., vol. 153, pp. 885-893, December 2006.

[11] X. Ma, J. Liu, and B. Bai, "New techniques for upper-bounding the MLD performance of binary linear codes," in Proc. 2011 IEEE Int. Symp. Inform. Theory, Saint-Petersburg, Russian Federation, August 2011.

[12] R. J. McEliece, "On the BCJR trellis for linear block codes," IEEE Transactions on Information Theory, vol. 42, pp. 1072-1092, July 1996.

[13] A. Vardy, "Trellis structure of codes," in Handbook of Coding Theory, V. S. Pless and W. C. Huffman, Eds. Amsterdam, The Netherlands: Elsevier, 1998, vol. 2.

[14] E. Agrell, "On the Voronoi neighbor ratio for binary linear block codes," IEEE Transactions on Information Theory, vol. 44, pp. 3064-3072, Novomber 1998. 